\title{
Automatic refinement and efficient solver for non linear dynamic structural problems
}

\author{
Ewen Biotteau - Anthony Gravouil - Antonius Lubrecht \\ Alain Combescure
}

Université de Lyon, CNRS, INSA Lyon

LaMCoS - UMR 5259, F-69621, Villeurbanne

\{ewen.biotteau, anthony.gravouil, ton.lubrecht,alain.combescure\}@insa-lyon.fr

ABSTRACT. This paper presents an adaptive strategy dedicated to non-linear transient dynamic problems. The spatial mesh is optimized to ensure the accuracy of the solution. Beginning from a coarse mesh, an error indicator is used to estimate the discretization error and new elements are created where the prescribed accuracy is not reached. A localized multigrid solver is used and the strategy is applied recursively until the local mesh size ensures that the discretization error is less than the prescribed accuracy. The spatial mesh is recreated at each time step.

RÉSUMÉ. Cet article présente une stratégie de résolution adaptative pour la dynamique transitoire non linéaire permettant l'optimisation du maillage spatial en fonction d'une précision requise du vecteur d'état. A partir d'un maillage grossier, un solveur multigrille localisé est utilisé. Un indicateur d'erreur est calculé afin de contrôler la précision de la solution sur le maillage courant et de nouveaux éléments sont crées de manière recursive et hiérarchique jusqu'à ce que la discrétisation de l'ensemble de la structure permette d'assurer la précision de la solution. Le maillage spatial est réévalué à chaque pas de temps.

KEYWORDS: automatic refinement, error indicators, multigrid, plasticity, dynamics.

MOTS-CLÉS : raffinement automatique, indicateurs d'erreur, multigrille, plasticité, dynamique.

DOI:10.3166/EJCM.19.141-152 @ 2010 Lavoisier, Paris 


\section{Introduction}

In design departments, engineers are interested in modeling problems which are more and more complex, accounting for dynamical aspects and non linear phenomena. The most common numerical technique used to discretize the space domain is the finite element method (FEM). A direct time integration scheme is frequently employed to describe the evolution of the structure. Such simulations require a large number of degrees of freedom (dof) in space and time to ensure the accuracy of the solution.

However, even if the computer capacity has increased dramatically over the last decades, some non linear dynamical solutions cannot be attained without efficient strategies which reduce $\mathrm{CPU}$ and memory requirement. A key point to efficiency is to use a good solver on an optimized mesh in space and time while assuring the precision.

For non linear problems, a large part of the computing time is spent in the resolution of the linearized equations. Direct and iterative solvers have shown their dependency on the number of degrees of freedom N. Using a solver with a linear convergence rate is a good way to ensure the reduction of the computing time. The multigrid method is known to have a complexity in $\mathcal{O}(N)$ for many simple problem (Brandt, 1977) and has soon shown its ability to solve non linear finite element problems (Kacou et al., 1993; Fish et al., 1995).

Various approaches have been developed to assess the precision of the solution. In adaptive procedures, a posteriori error estimates have been widely used (see references (Zhu et al., 1988; Babuska, 1978; Ladeveze et al., 2001)). However, the computation time of these error estimators is important. For specific applications, one can use error indicators which are cheaper in terms of $\mathrm{CPU}$ requirement even though they might be less accurate.

Adaptive procedures and space-time mesh optimization is an extensive field of research. For example, spatial adaptivity for elastoplastic behaviour, based on a multigrid strategy, is treated in (Ekevid et al., 2004). A domain decomposition method to couple sub-domains with their own numerical schemes and time steps is proposed in (Mahjoubi et al., 2009). A discontinuous Galerkin method working on unstructured space-time meshes is proposed in (Abedi et al., 2006). Cavin et al. present a fully automatic space-time refinement method based on classical and robust algorithms.

This paper extends the method proposed in (Cavin et al., 2005) and presents a localized multigrid strategy dedicated to non linear behaviour in transient dynamics. Its main specificity is that the refinement and the coarsening of the mesh between time steps is fully automatic and does not require human intervention. The first section introduces the reference problem and the numerical discretization. The following section sets up the multigrid solver and the localization strategy. The numerical performance of the solver is presented in Section 4. Section 5 concludes on the efficiency of the strategy and gives some perspectives. 


\section{Reference problem}

We consider the motion of a deformable body, assumed homogeneous and isotropic, occupying a domain $\Omega(t)$ with boundary $\partial \Omega(t)$ in the time interval $[0, T]$. Prescribed displacements $\underline{\mathbf{u}}_{d}(t)$ and traction forces per unit area $\underline{\mathbf{F}}_{d}(t)$ are applied on the subsets $\partial \Omega_{u}$ and $\partial \Omega_{f}$ of the boundary. $\underline{\mathbf{f}}(t)$ is a body force per unit volume.

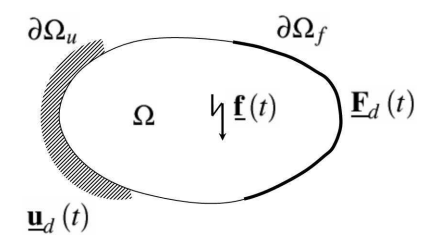

Figure 1. Reference continuous problem

The material is elastoplastic. The problem consists in the calculation of the displacement $\underline{\mathbf{u}}$, the internal variables $\nu_{i}$ and the stress tensor $\underline{\underline{\sigma}}$ under the assumption of small perturbations. One denotes $\mathcal{S}$ the complete state vector.

In non linear dynamics, using the FEM for the space discretization and the Newmark scheme for the time integration, the principle of virtual works leads to the discretized formulation presented Equation [1] at each time $t^{m}$. Lagrange multipliers $\boldsymbol{\Lambda}$ are used to impose the prescribed displacements.

$$
\begin{array}{r}
\mathbf{M} \ddot{\mathbf{U}}^{m}+\mathbf{F}_{\text {int }}^{m}=\mathbf{F}_{\mathrm{ext}}^{m}+\mathbf{L}^{\mathrm{T}} \boldsymbol{\Lambda}^{m} \\
\text { with: } \quad \mathbf{L} \mathbf{U}^{m}=\mathbf{U}_{d}
\end{array}
$$

$\mathbf{U}$ and $\ddot{\mathbf{U}}$ are the discretized displacement and acceleration vectors. $\mathbf{M}$ represents the mass matrix. $\mathbf{F}_{\text {ext }}$ and $\mathbf{F}_{\text {int }}$ are the external and internal force vectors. $\mathbf{L}$ is such that $\mathbf{U}$ matches with the prescribed displacement on the boundary. The problem consists of finding the state vector satisfying Equation [1] and the initial conditions :

$$
\left.\mathbf{U}\right|_{\mathrm{t}=0}=\mathbf{U}_{0},\left.\quad \dot{\mathbf{U}}\right|_{\mathrm{t}=0}=\dot{\mathbf{U}}_{0}
$$

The Newmark scheme provides Equations [3-4] to calculate the velocity and the displacement. The Newmark predictors ${ }^{\mathrm{p}} \mathbf{U}^{m}$ and ${ }^{\mathrm{p}} \dot{\mathbf{U}}^{m}$ are defined using the kinematic fields on the previous time step (Belytschko et al., 2005).

$$
\begin{gathered}
\mathbf{U}^{m+1}={ }^{\mathrm{p}} \mathbf{U}^{m}+\beta \Delta t^{2} \ddot{\mathbf{U}}^{m+1} \\
\dot{\mathbf{U}}^{m+1}={ }^{\mathrm{p}} \dot{\mathbf{U}}^{m}+\gamma \Delta t \ddot{\mathbf{U}}^{m+1}
\end{gathered}
$$

The constitutive law is integrated in each Gauss point by a radial return mapping (RRM) algorithm (Simo et al., 2000). The Cauchy stress tensor and the internal variables are updated and the complete state vector $\mathcal{S}$ is known on $t_{m}$. 


\section{Multigrid solver and localization strategy}

\subsection{Principle}

The strategy is initialized at each time step with the calculation of the state vector on two coarse hierarchical meshes $\mathcal{M}^{1}$ and $\mathcal{M}^{2}$. Then, an error indicator (see Section 3.4) is used to compare the solutions $\mathcal{S}^{1}$ and $\mathcal{S}^{2}$ on these meshes and new elements are created where the requested accuracy is not reached to form the mesh $\mathcal{M}^{3}$. Figure 2 illustrates the refinement method.

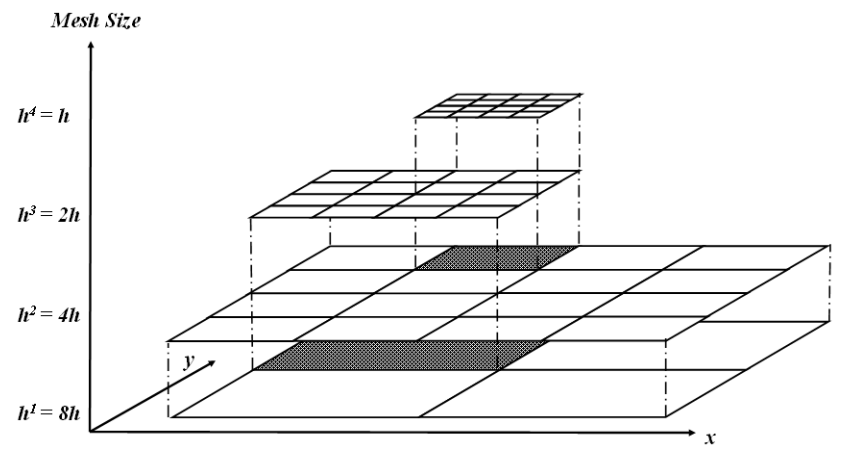

Figure 2. Bi-dimensional hierarchical refinement. Shaded elements do not verify the requested accuracy

One generalizes the explanation using the subscript $n$ for the current mesh level. If the new mesh $\mathcal{M}^{n+1}$ does not cover the entire mesh $\mathcal{M}^{n}$, one defines $\mathcal{M}_{+}^{n}$ and $\mathcal{M}_{*}^{n}$ as the covered and uncovered part of $\mathcal{M}^{n}$.

The restriction of the state vector $\mathcal{S}^{n}$ on $\mathcal{M}_{+}^{n}$ is interpolated in space on the mesh $\mathcal{M}^{n+1}$ as a first approximation. The calculation is performed on this new mesh using a multigrid solver and appropriate boundary conditions (see Section 3.2.4). Once the state vector $\mathcal{S}^{n+1}$ is known, the strategy is applied recursively and new meshes are eventually created. The process stops when the finest mesh verifies the requested accuracy on each of its elements.

\subsection{Notations and multigrid tools}

\subsubsection{Concerning multigrid}

The basic idea of multigrid methods is that iterative methods, such as conjugate gradient solvers, efficiently reduce the error components with wavelengths comparable to the grid size and only slowly reduce the large wavelength components. Using coarser meshes to solve these smooth error components, the multigrid procedure improves the convergence speed. 
Figure 3 illustrates the multigrid strategy in the case of four grids. The resolution is performed following the arrows. $\nu_{1}$ iterations of the solver are used to smooth the error before coarsening. $\nu_{2}$ iterations are made during the prolongation phase to remove the errors introduced in the interpolation. The gain in convergence speed highly depends on this two parameters. Using a Newton solver for the relaxation steps, experience shows that $\nu_{1}=\nu_{2}=1$ is the best choice. The problem is solved until convergence on the coarser mesh through $\nu_{0}$ iterations.

$\begin{array}{cc}\text { Mesh size } & \text { Level } \\ h^{4}=h & 4 \\ h^{3}=2 h & 3 \\ h^{2}=4 h & 2 \\ h^{1}=8 h & 1\end{array}$

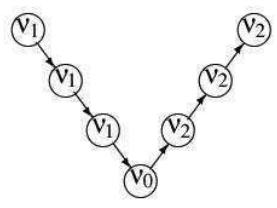

Figure 3. Flow diagram of a $V\left(\nu_{1}, \nu_{2}\right)$ cycle

All the meshes used in our multigrid strategy are hierarchical with a mesh size ratio of two. This choice is quasi optimal as it ensure a good compromise between the number of grids, local refinement flexibility and the error reduction in the V-cycle (Venner et al., 2000).

\subsubsection{Intergrid transfer}

For the prolongation of nodal fields, one uses the collocation procedure defined in (Dureisseix et al., 2006). The prolongation operator $\mathbb{I}_{n-1}^{n}$ between meshes $\mathcal{M}^{n-1}$ and $\mathcal{M}^{n}$ is constructed using the shape functions on level $(n-1)$ in the natural coordinate of the node belonging to level $n$.

If one denotes $\mathbf{X}^{n-1}$ the nodal field to transfer and $\left(\mathbf{X}_{j}^{n-1}, \mathbf{N}_{j}^{n-1}\right)$ the dof and the shape function of the node $j$ belonging to the mesh on level $(n-1)$, one obtains the dof of the node $i$ belonging to level $n$ with coordinates $\mathbf{M}_{i}^{n}$ :

$$
\mathbf{X}_{i}^{n}=\mathbf{X}_{j}^{n-1}{ }^{T} \mathbf{N}_{j}^{n-1}\left(\mathbf{M}_{i}^{n}\right)
$$

Once the prolongation operator is defined, the restriction operator $\mathbb{I}_{n}^{n-1}$ is built to ensure the energy conservation and one needs to impose the following relation:

$$
\mathbb{I}_{n}^{n-1}=\left(\mathbb{I}_{n-1}^{n}\right)^{T}
$$

The internal variables and the stress tensor are interpolated between the meshes using the shape functions of the elements. Then, a local least square method is used to compute the values in the Gauss points.

When one restricts the entire state vector (including both the nodal values and the Gauss point values) from level $n$ to $(n-1)$, the restriction operator is denoted $\mathcal{I}_{n}^{n-1}$ 


\subsubsection{Iterative solver}

The time subscript $m$ and the mesh level subscript $n$ are omitted in this section. One briefly presents the Newton solver used to reduce the residual defined by Equation [7] on each relaxation step in the multigrid process.

$$
\mathbf{R}=\mathbf{F}_{\text {ext }}+\mathbf{L}^{\mathrm{T}} \boldsymbol{\Lambda}-\mathbf{F}_{\text {int }}-\mathbf{M}^{m}=0
$$

Assuming that the Newton approximation and its associated residual on iteration $k$ are known, one linearizes Equation [7] using the order one Taylor expansion. This equation, to be solved in terms of $\Delta \mathbf{U}^{(k+1)}$, requires the Jacobian matrix.

The Jacobian matrix is recalculated at each Newton step. The modified Newton method considers the Jacobian matrix constant. Thus the number of iterations is higher but the cost of each one is less. The simplest choice consists in replacing the elastoplastic stiffness by the elastic one and one needs to solve at each Newton step:

$$
\begin{aligned}
& \mathbf{A} \Delta \mathbf{U}^{(k+1)}=\mathbf{R}^{(k)} \quad \text { with } \quad \mathbf{A}=\left(\mathbf{M}+\beta \Delta t^{2} \mathbf{K}\right) /\left(\beta \Delta t^{2}\right) \\
& \mathbf{U}^{(k+1)}=\mathbf{U}^{(k)}+\Delta \mathbf{U}^{(k+1)}
\end{aligned}
$$

Equation [9] is used to update the displacement vector. The complete state vector is updated using Equations [3] and [4] for the kinematic quantities and the $R R M$ algorithm for the elastoplastic state.

\subsubsection{Boundary conditions}

When a localized mesh $\mathcal{M}^{n}$ is created, it inherits boundary conditions from the closest coarser mesh. Displacements and forces are prescribed on the parts $\partial \mathcal{M}_{u}^{n}$ and $\partial \mathcal{M}_{f}^{n}$ of $\partial \mathcal{M}^{n}$ which intersect the boundaries $\partial \Omega_{u}$ and $\partial \Omega_{f}$ of the structure.

One defines $\partial \mathcal{M}_{l}^{n}$ as the complementary part on $\partial \mathcal{M}^{n}$ of the boundaries $\partial \mathcal{M}_{u}^{n}$ and $\partial \mathcal{M}_{f}^{n}$. This boundary marks the link between the meshes on level $n$ and $(n-1)$ and an other boundary condition is needed on this interface. The displacement of its nodes are imposed by the interpolation of the displacement field $\mathbf{U}^{n-1}$ using Lagrange multipliers.

\subsection{Multigrid solver}

We described the localized non linear multigrid strategy on level $\mathcal{M}^{n}$ used to compute the converged state vector $\mathcal{S}_{c}^{n}$. On each time step, one wants to solve Equation [1]. To simplify the explanation of the solver, this equation is summarized in Equation [10]. $\langle\bullet\rangle$ denotes the dependency of $\mathcal{A}$ on the state vector. One should refer to Figure 3 for better understanding.

$$
\mathcal{A}^{n}\left\langle\mathcal{S}^{n}\right\rangle=\mathbf{b}^{n}
$$


One supposes that an initial solution $\mathcal{S}^{n}$ is known (interpolated from $\mathcal{M}_{+}^{n-1}$ or after a few multigrid cycles). After $\nu_{1}$ iterations, one obtains an approximation $\tilde{\mathcal{S}}^{n}$ of the solution. This state vector is associated with an error $v^{n}$ and a residual $\mathbf{R}^{n}$.

$$
\mathcal{A}^{n}\left\langle\mathcal{S}_{c}^{n}\right\rangle=\mathbf{b}^{n} \Rightarrow \mathcal{A}^{n}\left\langle\tilde{\mathcal{S}}^{n}+v^{n}\right\rangle=\mathcal{A}^{n}\left\langle\tilde{\mathcal{S}}^{n}\right\rangle+\mathbf{R}^{n}
$$

During the coarsening phase, if $\mathcal{M}^{n}$ does not cover the entire mesh $\mathcal{M}^{n-1}$, one builds the state vector on level $(n-1)$ using the restriction of $\tilde{\mathcal{S}}^{n}$ on $\mathcal{M}_{+}^{n-1}$ and the restriction of the previous state vector $\mathcal{S}^{n-1}$ on $\mathcal{M}_{*}^{n-1}$. If one denotes $\Pi_{*}^{n-1}$ and $\Pi_{+}^{n-1}$ the restriction operators on these meshes, the current state vector $\hat{\mathcal{S}}^{n-1}$ is defined by:

$$
\hat{\mathcal{S}}^{n-1}=\Pi_{*}^{n-1} \mathcal{S}^{n-1}+\Pi_{+}^{n-1} \mathcal{I}_{n}^{n-1} \tilde{\mathcal{S}}^{n}
$$

Where the operator + denotes the concatenation operator. The calculation on level $(n-1)$ is performed to approximate the error $v^{n}$ on level $(n-1)$. Equation [13] defines the equivalent problem on the mesh $\mathcal{M}^{n-1}$ :

$$
\mathcal{A}^{n-1}\left\langle\hat{\mathcal{S}}^{n-1}+v^{n-1}\right\rangle=\mathcal{A}^{n-1}\left\langle\hat{\mathcal{S}}^{n-1}\right\rangle+\mathbb{I}_{n}^{n+1} \mathbf{R}^{n}
$$

If the mesh $\mathcal{M}^{n-1}$ contains too many dofs, solving the problem until convergence could be expensive. That is why one uses recursive multigrid cycles to reduce the number of dofs of the coarsest mesh and obtain a cheap problem to solve until convergence. Whatever the calculation strategy on $\mathcal{M}^{n-1}$, a new approximate solution $\tilde{\mathcal{S}}^{n-1}$ is obtained.

As plasticity is an irreversible phenomenon, the prolongation of the correction is not as simple as the coarsening phase. Interpolating the entire state vector from $\mathcal{M}^{n-1}$ to $\mathcal{M}^{n}$ may introduce irreversible errors. Then, during the prolongation phase one uses the system of Equation [14-17] to correct the solution $\tilde{\mathcal{S}}^{n}$ :

$$
\begin{aligned}
\mathbf{U}^{n} & =\tilde{\mathbf{U}}^{n}+\mathbb{I}_{n-1}^{n}\left(\tilde{\mathbf{U}}^{n-1}-\hat{\mathbf{U}}^{n-1}\right) \\
\ddot{\mathbf{U}}^{n} & =\left(1 / \beta \Delta t^{2}\right) \cdot\left(\mathbf{U}^{n}-{ }^{\mathrm{p}} \mathbf{U}^{n}\right) \\
\dot{\mathbf{U}}^{n} & ={ }^{\mathrm{p}} \dot{\mathbf{U}}+\gamma \Delta t \ddot{\mathbf{U}}^{n} \\
\left(\boldsymbol{\sigma}^{n}, \nu_{i}^{n}\right) & =\operatorname{RRM}\left(\left(\mathbf{U}^{n}-\tilde{\mathbf{U}}^{n}\right), \tilde{\boldsymbol{\sigma}}^{n}, \tilde{\nu}_{i}^{n}\right)
\end{aligned}
$$

Once the correction is prolongated on the mesh $\mathcal{M}^{n}$, a few iterations $\nu_{2}$ is performed and new multigrid cycles are performed until convergence.

Figure 4 illustrates the interpolation and restriction operations between the meshes in the multigrid cycle. The calculation begins on the coarser level as described in Section 3.1. In transient dynamics, one needs to interpolate in time the state vector to compute the predictors of the Newmark scheme and the previous elastoplastic state of the structure. When the calculation is performed for the first time on a mesh, the non linear state is updated from the converged mesh on the previous time step. 


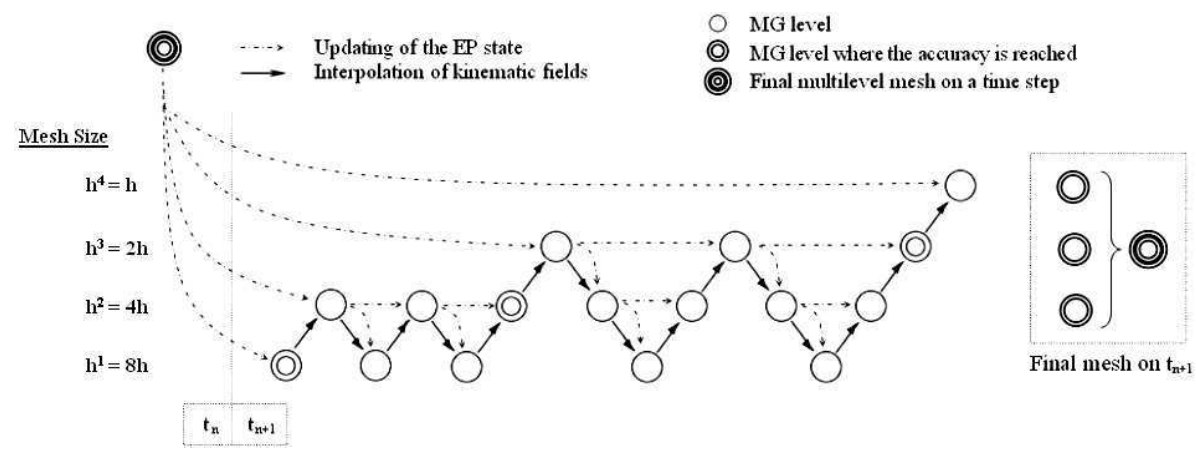

Figure 4. Interpolation and updating of the state vector among space and time

\subsection{Error indicator}

To determine the area where a finer mesh is needed, one uses error indicators. At each time $t_{m}$, the solutions on two successive meshes are compared to evaluate the discretization error. If the problem is regular, one obtains an estimation of the accuracy of the solution.

Various indicators can be defined depending on the part of the state vector which is used in the post-processing. We limit this section to the definition of the energy error indicator used in Section 4.

One denotes $e_{j}^{n-1}$ the element $j$ on level $(n-1)$ and $\sum e_{i}^{n}$ the matching hierarchical elements on level $n$. $\left.\bullet\right|_{e}$ denotes the restriction on $e$. If $\mathcal{E}\left(t_{m}\right)$ defines the energy on the structure at time $t_{m}$, including both the internal and the kinetic contribution, one gets the error indicator on the element $e_{j}^{n-1}$ on Equation [18] at each time $t_{m}$.

$$
\left.\boldsymbol{\epsilon}_{\mathcal{E}}\right|_{e_{j}^{n-1}}=\frac{\sqrt{\left|\left(\left.\mathcal{E}^{n-1}\left(t_{m}\right)\right|_{e_{j}^{n-1}}-\left.\mathcal{E}^{n}\left(t_{m}\right)\right|_{\sum e_{i}^{n}}\right)\right|}}{\max _{[0 ; T]} \sqrt{\mathcal{E}^{1}(t)}}
$$

The use of the square root defines a norm. One divides by the maximum on the coarsest level to obtain a relative error indicator. This indicator is defined on each element of the mesh $\mathcal{M}_{+}^{n-1}$. The automatic refinement process is driven by the requested accuracy and finer levels are created when the error exceeds the criterion.

\section{Results}

The following examples were calculated using Castem 2000 (Verpeaux et al., 1991). The material is assumed to be linear elastic plastic (positive isotropic hardening) with a Young modulus equal to $210 \cdot 10^{9} \mathrm{~Pa}$, a density of $7800 \mathrm{~kg} \cdot \mathrm{m}^{-3}$, a plastic stiffness of $21 \cdot 10^{9} \mathrm{~Pa}$ and a yield stress of $300 \cdot 10^{6} \mathrm{~Pa}$. 
As an example, we use a clamped beam under a traction force. Plasticity occurs when the wave front arrives on the clamped end. The mean acceleration scheme $(\gamma=$ $0.5, \beta=0.25)$ is used.

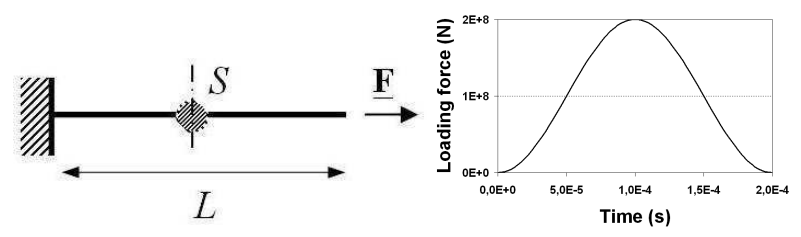

$\begin{array}{ll}\text { (a) } 1 \mathrm{D} \text { example - Clamped beam } & \text { (b) Loading force }\end{array}$

Figure 5. One-dimensional problem of a beam under a traction force

\subsection{Convergence rate}

In this section, no localization is allowed and the meshes cover the entire structure. The multigrid cycle consists of 4 meshes. The coarser one has 128 elements and the finest one 1024 .

Figure 6 shows the relative residual norm as a function of the relative number of iterations on the finest mesh. The use of multigrid strategies improves the convergence rate of the modified Newton solver $(N R S)$.

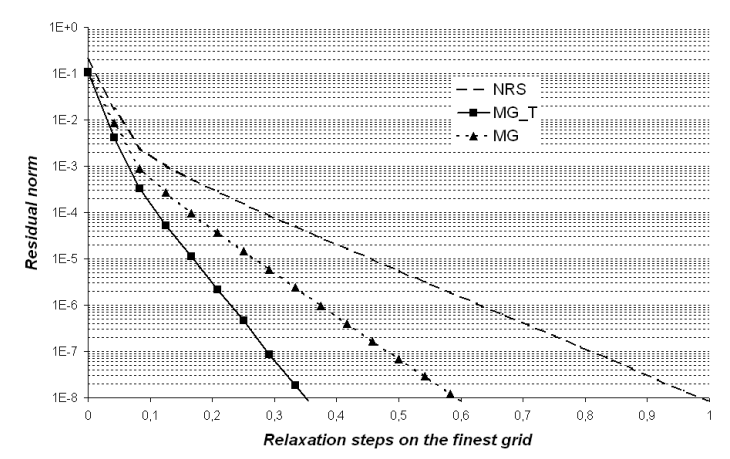

Figure 6. Residual norm as a function of the number of iteration on the finest level

The choice of the restricted quantities during the coarsening phase changes the convergence rate of the non linear multigrid solver. $M G \_T$ denotes the multigrid strategy described on Section 3.3 with the complete state vector coarsening. $M G$ denotes a multigrid strategy where the elastoplastic state is independent on each mesh (kinematic restriction only, the material state is updated using the $R R M$ algorithm).

The convergence rate of $M G$ exceeds that of $M G \_T$. In fact, plasticity might be to localized to be detected and computed on the coarser meshes. As a result, the 
plastic state is different on each mesh if one does not coarsen the complete non linear state vector and the efficiency of the multigrid strategy is reduced.

Moreover, in the case where the finest mesh $\mathcal{M}^{n}$ is localized, reducing the complete state vector on $\mathcal{M}_{+}^{n-1}$ allows to update the state vector on $\mathcal{M}_{*}^{n-1}$ as long as a localized non linearity influences the entire structure.

\subsection{One-dimensional automatic refinement}

Figure 7 highlights the behaviour of the automatic refinement method. Space-time diagrams are used, the horizontal axis represents time while the vertical one represents space. The coarsest level has 16 elements and the time step is $d t=1.1 \mu s$. The nodes of the meshes are plotted every 8 time steps. The impact occurs on the upper part of the beam on time $t=0$. The energy indicator defined by Equation [18] is used with a requested accuracy of $1 \cdot 10^{-2}$.

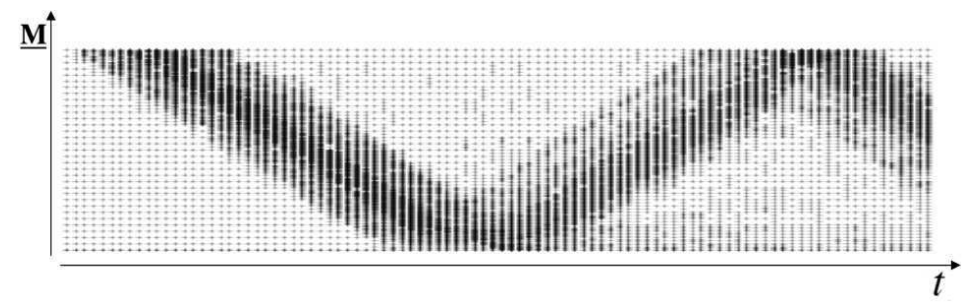

Figure 7. Space-time diagrams of the one-dimensional beam using an energy error indicator and a requested accuracy of $1 \cdot 10^{-2}$

Using the localized multigrid strategy, spatial coarsening occurs without specific techniques because the mesh is reassessed at each time step. The refinement follows the wave propagation in the beam. Finer levels are created when the calculation is not accurate enough and the refinement occurs in the maximum energy area at each time step. The mesh remains fine on the clamped end of the beam when plasticity occurs to describe the gradient in plastic strain and the energy contribution due to plastic strain.

As the indicator focuses on the energy error, the accuracy in plastic strain is not ensured. If the plastic contribution in energy is small compared to the elastic one, the refinement focuses on the elastic part. The use of a plastic work indicator ensures the accuracy of the non linear fields but neglects the elastic part.

\subsection{Precision}

The numerical example presented Section 4.2 is used with different requested accuracies. A reference solution $\mathcal{S}^{r}$ is calculated on 32768 elements corresponding to a uniform mesh size of level 12 . 
The first two columns of Table 1 present the requested accuracy and the time average of the error maximum over the structure. To avoid the influence of the time discretization, the error is defined as the difference between the increment in energy on the reference and on the converged mesh of the localized multigrid solver. This table shows the correlation between the requested accuracy and the obtained error in energy. It can be concluded from this table that the requested accuracy is indeed obtained.

Table 1. Accuracy in energy and gain in elements for the one-dimensional problem

\begin{tabular}{cccc}
\hline $\begin{array}{c}\text { Requested } \\
\text { accuracy }\end{array}$ & $\begin{array}{c}\text { Maximum } \\
\text { error }\end{array}$ & $\begin{array}{c}\text { Number } \\
\text { of meshes }\end{array}$ & $\begin{array}{c}\text { Relative number } \\
\text { of elements }(\%)\end{array}$ \\
\hline $5 \cdot 10^{-2}$ & $2.19 \cdot 10^{-2}$ & 6 & 16.3 \\
$2 \cdot 10^{-2}$ & $1.16 \cdot 10^{-2}$ & 7 & 14.3 \\
$1 \cdot 10^{-2}$ & $7.92 \cdot 10^{-3}$ & 8 & 12.3 \\
\hline
\end{tabular}

The maximum number of meshes used in the multigrid strategy over the time is given in column three. The last column shows the gain in elements comparing the time average number of elements in the multigrid strategy and the number on a uniform mesh with the same mesh size as the maximum level reached.

\section{Conclusion}

This paper presents an adaptive strategy dedicated to non linear transient dynamic simulations. The strategy uses a multigrid solver on each time step. The state vectors on successive space meshes are compared and a relative error indicator is associated with each element of the mesh. The automatic refinement process is driven by a requested accuracy of this indicator. The spatial mesh is reassessed on each time step and the coarsening occurs without specific techniques.

The strategy is illustrated using the example of an elastoplastic beam with isotropic hardening. Using an energy error indicator, one observes that the mesh refinement follows the physical phenomena: the refinement occurs in the wave propagation zone and the mesh remains fine in the plastic zone to describe the plastic contribution.

It was shown that the convergence rate of the iterative solver increases using the appropriate strategy for the coarsening phase. Moreover, using the localization strategy, it is shown that the number of elements is reduced for a given accuracy of the state vector. Then, the calculation of the state vector over the time interval is performed within acceptable calculation time. This gain is expected to be higher for problems in higher dimensions.

Further work will investigate the influence of the error indicators on the behaviour of the refinement method, and the strategy should be extended to space-time refinement. 


\section{References}

Abedi R., Haber R., Thite S., Erickson J., “ An h-adaptive Spacetime-Discontinuous Galerkin Method for Linearized Elastodynamics", European Journal of Computational Mechanics, vol. $15, n^{\circ} 6$, p. 619-642, 2006.

Babuska I., " A posteriori error estimates for the finite element method", International Journal for Numerical Methods in Engineering, vol. 12, n 10, p. 1597-1615, 1978.

Belytschko T., Liu W. K., Moran B., Non linear finite elements for continua and structures, Wiley, New York, 2005.

Brandt A., "Multilevel Adaptive Solutions to boundary value problems", Mathematics of Computation, vol. $31, \mathrm{n}^{\circ} 138$, p. 333-390, 1977.

Cavin P., Gravouil A., Lubrecht A. A., Combescure A., “ Automatic energy conserving spacetime refinement for linear dynamic structural problems", International Journal for Numerical Methods in Engineering, vol. 64, n 3, p. 304-321, 2005.

Dureisseix D., Bavestrello H., " Information transfer between incompatible finite element meshes: Application to coupled thermo-viscoelasticity", Computer Methods in Applied Mechanics and Engineering, vol. 195, n 44-47, p. 6523 - 6541, 2006.

Ekevid T., Kettil P., Wiberg N. E., " Adaptive multigrid for finite element computations in plasticity", Computers and Structures, vol. 82, n 28, p. 2413 - 2424, 2004.

Fish J., Pandheeradi M., Belsky V., “ An efficient multilevel solution scheme for large scale non-linear systems", International Journal for Numerical Methods in Engineering, vol. 38, $\mathrm{n}^{\circ} 10$, p. 1597-1610, 1995.

Kacou S., Parsons I. D., " A parallel multigrid method for history-dependent elastoplacticity computations", Computer Methods in Applied Mechanics and Engineering, vol. 108, $\mathrm{n}^{\circ} 1$ 2, p. 1-21, 1993.

Ladeveze P., Pelle J. P., La maitrise du calcul en mecanique lineaire et non lineaire, Hermes Sciences Publications, Paris, 2001.

Mahjoubi N., Gravouil A., Combescure A., "Coupling subdomains with heterogeneous time integrators and incompatible time steps", Computational Mechanics, vol. 44, n ${ }^{\circ}$ 6, p. 825 $843,2009$.

Simo J. C., Hughes T. J. R., Computational inelasticity, Springer-Verlag, New York, 2000.

Venner C. H., Lubrecht A. A., Multilevel methods in lubrification, Tribology Series 37; Elsevier, Amsterdam, 2000.

Verpeaux P., Charras T., Millard A., Castem 2000 guide du developpement, Rapport dmt, Commissariat à l'Energie Atomique, 1991.

Wiberg N. E., Li X., " Adaptive finite element procedures for linear and non-linear dynamics", International Journal for Numerical Methods in Engineering, vol. 46, $\mathrm{n}^{\circ}$ 10, p. 1781-1802, 1999.

Zhu J. Z., Zienkiewicz O. C., " Adaptive techniques in the finite element method”, Communications in Applied Numerical Methods, vol. 4, $\mathrm{n}^{\circ}$ 2, p. 197-204, 1988. 